Supplementary information for

\title{
Surface Reconstruction of Halide Perovskites during Post-treatment
}

Shaun Tan, Tianyi Huang, Ilhan Yavuz, Rui Wang, Marc H. Weber, Yepin Zhao, Maged

Abdelsamie, Michael E. Liao, Hao-Cheng Wang, Kenny Huynh, Kung-Hwa Wei, Jingjing Xue,

Finn Babbe, Mark S. Goorsky, Jin-Wook Lee, ${ }^{*}$ Carolin M. Sutter-Fella,* and Yang Yang*

*Correspondence to:

Y. Yang, email: yangy@ucla.edu

C. M. Sutter-Fella, email: csutterfella@lbl.gov

J.-W. Lee, email: jw.lee@skku.edu

\section{Table of contents}

Materials and Methods

Figure S1. Photographs of ammonium salts in CF.

Figure S2. PL penetration depth estimation.

Figure S3. In situ and time-resolved PL spectra of the perovskite films.

Figure S4. PAS profile of the perovskite films.

Figure S5. Device energy alignment and performance.

Figure S6. Humidity stability testing on the perovskite films.

Figure S7. Modified surface treatment process.

Figure S8. In situ PL of films undergoing surface treatment.

Figure S9. In situ PL of films undergoing post-annealing. 


\section{Materials and Methods}

Film fabrication

All materials were purchased from Sigma-Aldrich, unless stated otherwise. The reference perovskite is based on a $\mathrm{FAPbI}_{3}$ composition with 5 mol \% of $\mathrm{MAPbBr}_{3}$ fabricated by a one-step antisolvent quenching method. $889 \mathrm{mg} \mathrm{mL}^{-1} \mathrm{FAPbI}_{3}$ (FAI: GreatCell Solar, $\mathrm{PbI}_{2}$ : TCI America), $33 \mathrm{mg} \mathrm{mL}{ }^{-1} \mathrm{MAPbBr}_{3}$ (MABr: 1-Material, $\mathrm{PbBr}_{2}$ : Alfa Aesar Chemicals), and $33 \mathrm{mg} \mathrm{ml}^{-1} \mathrm{MACl}$ (GreatCell Solar) were dissolved in a dimethylformamide (DMF)/dimethylsulfoxide (DMSO) mixed solvent $(8: 1 \mathrm{v} / \mathrm{v})$. The perovskite was deposited at $4000 \mathrm{rpm}$ for $20 \mathrm{~s}$, where $0.2 \mathrm{~mL}$ of diethyl ether was dropped after $10 \mathrm{~s}$, followed by annealing at $150{ }^{\circ} \mathrm{C}$ for $10 \mathrm{~min}$. Subsequently, for the treated films, pure (blank) IPA was dropped on a film while spinning at $5000 \mathrm{rpm}$, followed by annealing at $100{ }^{\circ} \mathrm{C}$ for $5 \mathrm{~min}$. The control films also underwent the same annealing at $100{ }^{\circ} \mathrm{C}$ for $5 \mathrm{~min}$.

\section{Device fabrication}

Indium tin oxide (ITO) on glass was cleaned by successive ultrasonication in detergent, deionized water, acetone, and isopropyl alcohol for $20 \mathrm{~min}$ each. The cleaned substrate was treated by ultraviolet ozone for $20 \mathrm{~min}$. $\mathrm{SnO}_{2}$ colloidal solution (Alfa Aesar Chemicals) was diluted in water (deionized) in 1:5 ratio and spun at $3000 \mathrm{rpm}$, followed by annealing at $165{ }^{\circ} \mathrm{C}$ for $35 \mathrm{~min}$. The prepared $\mathrm{SnO}_{2}$ film was treated with $10 \mathrm{mM} \mathrm{KOH} \mathrm{(aq)} \mathrm{at} 3000 \mathrm{rpm}$, followed by annealing at 100 ${ }^{\circ} \mathrm{C}$ for $10 \mathrm{~min}$. The active layer is deposited in the same procedure as described above. For $\mathrm{OABr}$ treatment, $15 \mathrm{mM} \mathrm{OABr}$ in $\mathrm{CF}$ was dropped on a film while spinning at $5000 \mathrm{rpm}$, followed by annealing at $100{ }^{\circ} \mathrm{C}$ for $5 \mathrm{~min}$. Subsequently, spiro-MeOTAD solution $(85.8 \mathrm{mg}$ of spiroMeOTAD (p-OLED Corp) in $1 \mathrm{~mL}$ of chlorobenzene with $33.8 \mu \mathrm{l}$ of 4-tert-butylpyridine and $19.3 \mu \mathrm{l} \mathrm{of} \mathrm{Li-TFSI} \mathrm{(520} \mathrm{mg} \mathrm{mL}^{-1}$ in acetonitrile) solution) was dropped at $3000 \mathrm{rpm}$ for $30 \mathrm{~s}$. For the top electrode, $80 \mathrm{~nm}$ gold was thermally deposited at an evaporation rate of $0.5 \mathrm{~A} \mathrm{~s}^{-1}$. For the anti-reflection coating, $150 \mathrm{~nm} \mathrm{MgF}_{2}$ was thermally deposited at an evaporation rate of $1 \mathrm{~A} \mathrm{~s}^{-1}$. Computational details

First-principles calculations were performed based on the density functional theory (DFT) using a plane-wave basis set and the projected augmented wave (PAW) method, as implemented in the VASP package. ${ }^{29,30}$ The $2 \times 2 \times N$ perovskite slabs were generated along the [001] direction and a 10-15 Å vacuum slab were added. For all geometry optimizations and self-consistent field calculations Perdew-Burke-Ernzerhof type generalized gradient approximation (GGA-PBE) ${ }^{31}$ for 
the exchange-correlation functional with a $300 \mathrm{eV}$ plane-wave cutoff and 4x4x1 k-point mesh for Brillouin zone sampling were chosen. Dispersion corrections to electronic energies based on Grimme's DFT-D3(BJ) scheme ${ }^{32,33}$ were included. Ionic positions and volumes were relaxed using a conjugate gradient algorithm, until all residual forces are smaller than $0.01 \mathrm{eV} / \AA$.

Computational formation energies are defined as:

$$
\Delta H(\operatorname{slab} / X)=H(\text { slab } / X)-H(\text { slab })-H(X)
$$

where $\quad$ slab $=\{$ Pristine, Reconstructed $\}$ represents different slabs and $X=$ $\{O A I, P E A I, O A B r\} . H($ slab $/ X), H($ slab $)$ and $H(X)$ is the total energy of the complex, clean slab and ligand, respectively. Computational ionization energies (IE) are obtained from the bandedge valence band maximum and plane-averaged electrostatic potentials (ESP):

$$
V(z)=\frac{1}{A} \iint V(x, y, z) d x d y
$$

ESP is used to determine the vacuum level $\left(E_{\mathrm{vac}}\right)$. Therefore, the IE is calculated from:

$$
I E=E_{v a c}-E_{v b m} .
$$

The surface energies for the pristine and reconstructed surfaces are calculated from the following expression:

$$
\gamma=\gamma^{c l}+\gamma^{r e l}
$$

where $\gamma^{c l}$ is the cleavage energy to form the clean slab, $\gamma^{r e l}$ is the surface energy change due to relaxation of the surface. $\gamma^{\text {cleave }}$ and $\gamma^{\text {rel }}$ values were obtained from:

$$
\gamma^{c l}=\frac{G_{\text {slab }}^{\text {clean }}-N G_{\text {bulk }}+\sum n_{i} \mu_{i}}{2 A} \text { and } \gamma^{\text {rel }}=\frac{G_{\text {slab }}^{\text {relax }}-G_{\text {slab }}^{\text {clean }}}{A}
$$

where $G_{b u l k}$ is the bulk energy per formula unit of the material, $\mathrm{N}$ is the number of sufficient bulk $\mathrm{FAPbI}_{3}$ units, $G_{\text {slab }}^{\text {clean }}$ is the energy of the clean slab, $G_{\text {slab }}^{\text {relax }}$ is the energy of the relaxed slab, $\mu_{i}$ is the chemical potential and $\mathrm{A}$ is the surface area.

\section{Positron Annihilation Spectroscopy}

Positron annihilation spectroscopy (PAS) was carried out at the Washington State University positron beam. Monoenergetic positrons with energies selected from 0.02 to $70 \mathrm{keV}$ are transported with a $\sim 180$ gauss (0.018 Tesla) guiding field. The isotope ${ }^{22} \mathrm{Na}$ is the source of positrons some of which moderated with a $\mathrm{W}$ thin film single crystal moderator to feed the beam. In the target sample positrons thermalize within $\sim 5$ ps at a mean depth that is governed by the incident kinetic energy and the density of the material. ${ }^{34}$ Annihilations predominantly occur into two near colinearly emitted photons with an average energy of $511 \mathrm{keV}$ each. A high purity high 
efficiency Ge detector with an energy resolution of $1.4 \mathrm{keV}$ with full-width-at-half-maximum (FWHM) at $511 \mathrm{keV}$ is used to detect and energy analyze the deposited energy of the photons. Only events in the photoelectric peaks are considered for the Doppler broadening measurements. The data in the photoelectric peak are area normalized to unit total counts after suitable background subtraction. The fraction of events collected in a narrow $1.490 \mathrm{keV}$ wide region centered at 511 $\mathrm{keV}$ and the two wing regions from $2.61 \mathrm{keV}$ to $5.87 \mathrm{keV}$ on each side from the $511 \mathrm{keV}$ peak are extracted. They are the shape (S) and wing (W) parameters, respectively. Data analysis is performed with the standard Doppler broadening software called VepFit. ${ }^{35}$ Inputs are the values for $S$ with uncertainties at the measured energies. Experiments are carried out in dark and under vacuum. Care is taken to minimize effects of correlation between layer diffusion lengths and the thickness of the layer.

\section{Material and device characterizations}

In situ photoluminescence (PL) measurements were acquired during spin coating in a $\mathrm{N}_{2}$ glovebox with a home-built setup as described in previous works ${ }^{10,11}$ using a $405 \mathrm{~nm}$ laser diode, a planoconvex lens above the substrate, a $409 \mathrm{~nm}$ long-pass filter, and a fiber coupled Ocean Optics spectrometer (Flame) calibrated by the manufacturer. A maximum power density of $\sim 100$ $\mathrm{mW} / \mathrm{cm}^{2}$ was used during in situ PL measurements. Spectra were continuously acquired every 100 ms. PL emission spectra were fitted by using single Gaussian peak fitting after linear background removal and Jacobian transformation. ${ }^{36}$ The time resolved photoluminescence spectra (TRPL) were measured using a Picoharp 300 with time-correlated single-photon counting capabilities excited by the $640 \mathrm{~nm}$ pulse laser diode (PLD 800B, PicoQuant) with a repetition frequency of $200 \mathrm{kHz}$. X-ray/Ultraviolet Photoelectron Spectroscopy (XPS/UPS) measurements were carried out on an XPS AXIS Ultra DLD (Kratos Analytical). An Al Ka (1,486.6 eV) X-ray was used as the excitation source. A high-resolution Jordan Valley D1 X-ray diffractometer with $\mathrm{Cu} \mathrm{K} \alpha$ radiation and incident parallel beam optics was employed to obtain the $\omega: 2 \theta$ scans. The current density and voltage curves of the devices were measured with a Keithley 2401 source meter under simulated one sun AM 1.5G spectrum illumination $\left(100 \mathrm{~mW} \mathrm{~cm}^{-2}\right)$ from an Oriel Sol3A class AAA solar simulator (Newport). The light intensity was first calibrated with a NREL-certified Si photodiode with a KG-5 filter. All devices were measured in ambient air with a $0.100 \mathrm{~cm}^{2}$ sized metal aperture used to precisely define the active area during measurement. Devices were measured at a scan rate of $0.1 \mathrm{~V} \mathrm{~s}^{-1}$ from $1.2 \mathrm{~V}$ to $-0.1 \mathrm{~V}$ or $0.1 \mathrm{~V}$ to $1.2 \mathrm{~V}$ in $0.02 \mathrm{~V}$ steps. 


\section{Grazing Incidence X-ray Diffraction penetration depth estimation}

The penetration depths were estimated based on the equation: ${ }^{1}$

$$
G=\frac{\int_{x=0}^{x=x} d I_{D}}{\int_{x=0}^{x=\infty} d I_{D}}=1-\exp \left\{-\mu x\left(\frac{1}{\sin \gamma}+\frac{1}{\sin \beta}\right)\right\}
$$

where $\mathrm{x}$ is the penetration depth, $\gamma$ is the incident beam angle, $\beta$ is the exit beam angle, $\mu$ is the mass absorption coefficient $\left(207 \mathrm{~cm}^{2} \mathrm{~g}^{-1}\right.$ for $\left.\mathrm{FAPbI}_{3}\right)$ and $\mathrm{G}$ is a constant with value 0.95 . The above equation is solved to give the penetration depth $\mathrm{x}$. 


\section{Supplementary Figures}

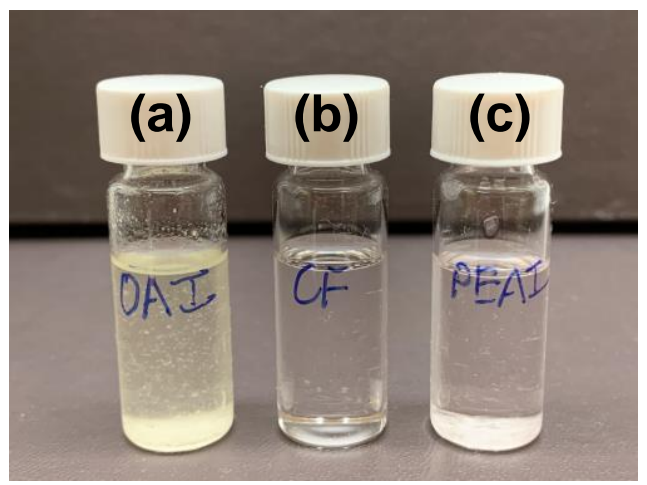

Figure S1. Photographs of ammonium salts in CF. Photographs of (a) $30 \mathrm{mmol}$ OAI in CF, (b) pure CF, and (c) 30 mmol PEAI in CF. OAI formed a suspension in CF while PEAI remained undissolved at the bottom of the vial in (c).

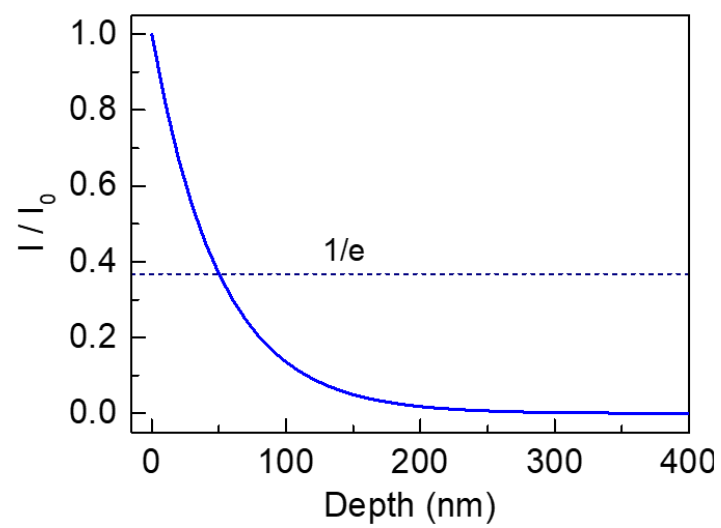

Figure S2. PL penetration depth estimation. Intensity decay calculated using the Beer-

Lambert law. The absorption coefficient is assumed to be $2 \times 10^{5} \mathrm{~cm}^{-1}$ as was reported for $\alpha$ $\mathrm{FAPbI}_{3}$ thin films. ${ }^{2}$ The penetration depth is defined as the depth by which the intensity decays to $\frac{1}{e}$ of its initial value. 
a

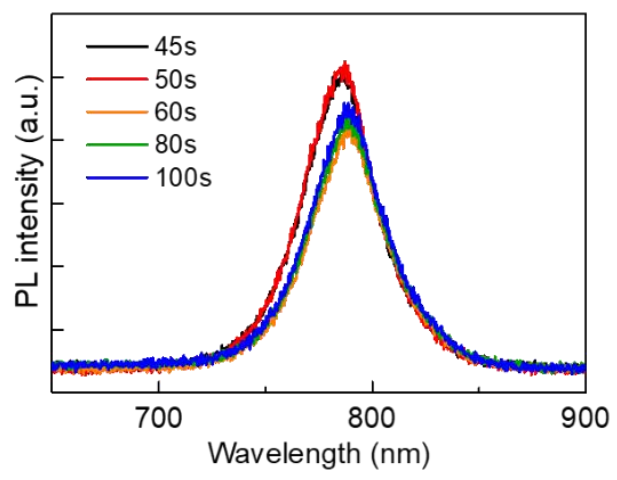

C

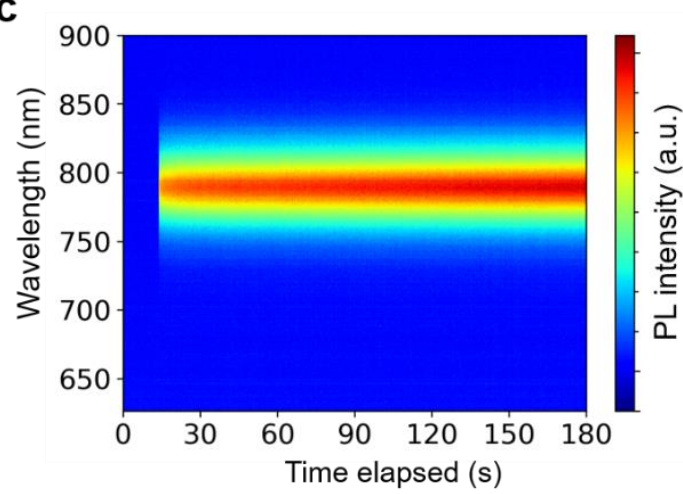

b

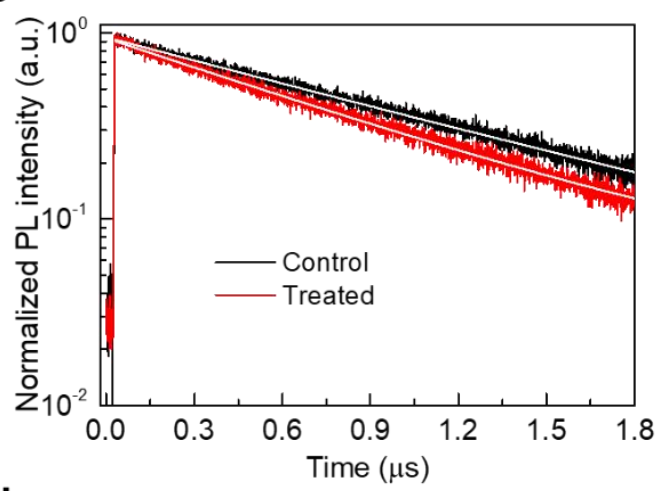

d

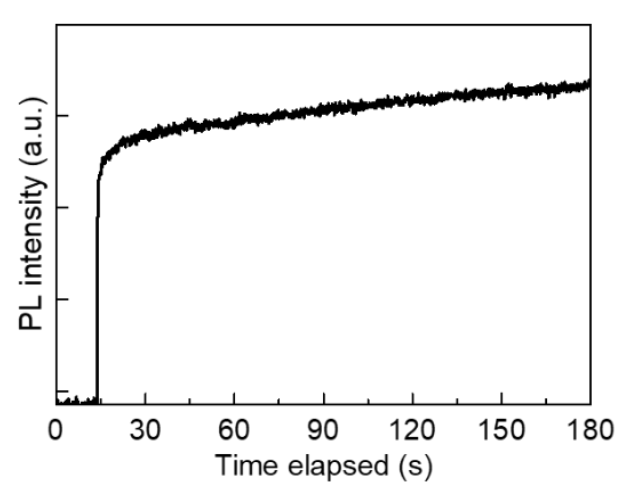

Figure S3. In situ and time-resolved PL spectra of the perovskite films. (a) Evolution of the raw PL spectra of the perovskite film undergoing treatment with IPA. (b) Normalized timeresolved PL spectra of the perovskite films on glass. Solid white lines are the fitted profiles using a mono-exponential decay function. (c) Contour plot and (d) evolution of the PL intensity of a perovskite film with time. 
a

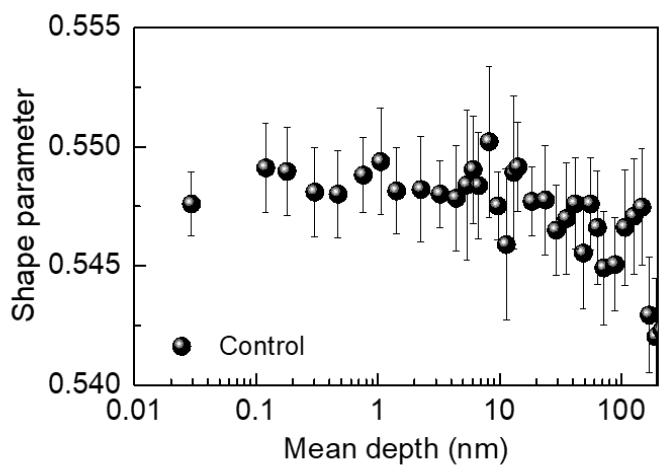

b

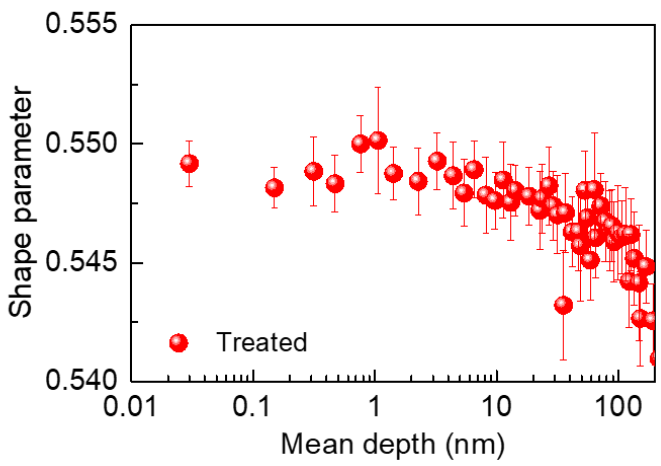

Figure S4. PAS profile of the perovskite films. PAS depth-profiling of the (a) control and (b) treated perovskite films. 

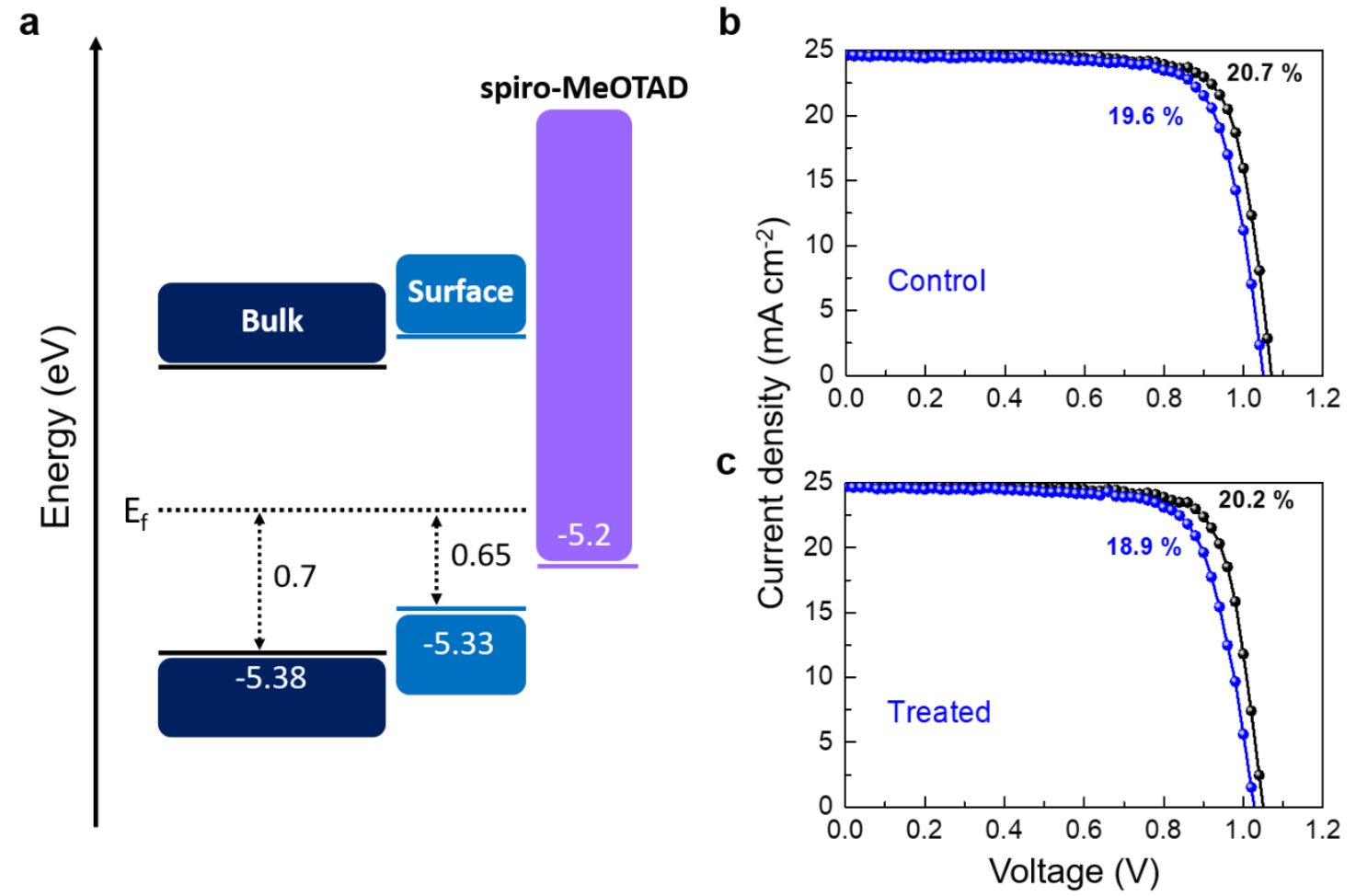

Figure S5. Device energy alignment and performance. (a) Schematic band diagram of the energy levels of the IPA treated perovskite film relative to the fermi level. Current densityvoltage curves of the (b) control and (c) treated devices in reverse (in black) and forward (in blue) scans.

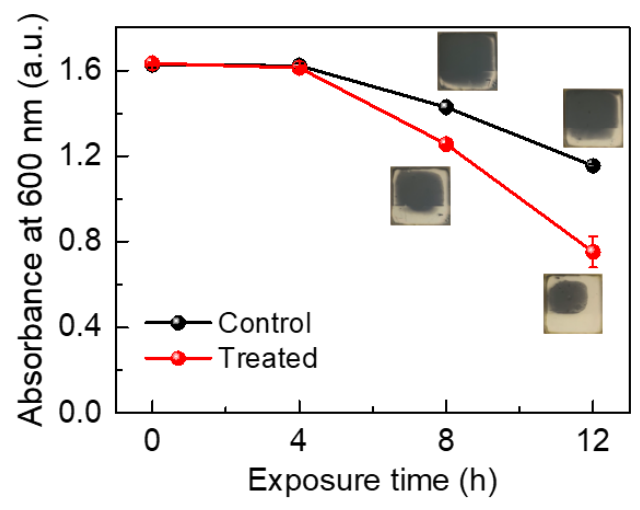

Figure S6. Humidity stability testing on the perovskite films. Absorbance at $600 \mathrm{~nm}$ of the perovskite films exposed to RH $75 \pm 10 \%$ with time. Inset shows photographs of the films. 
a

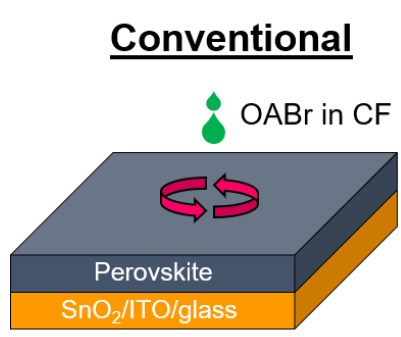

b

Modified 2-step
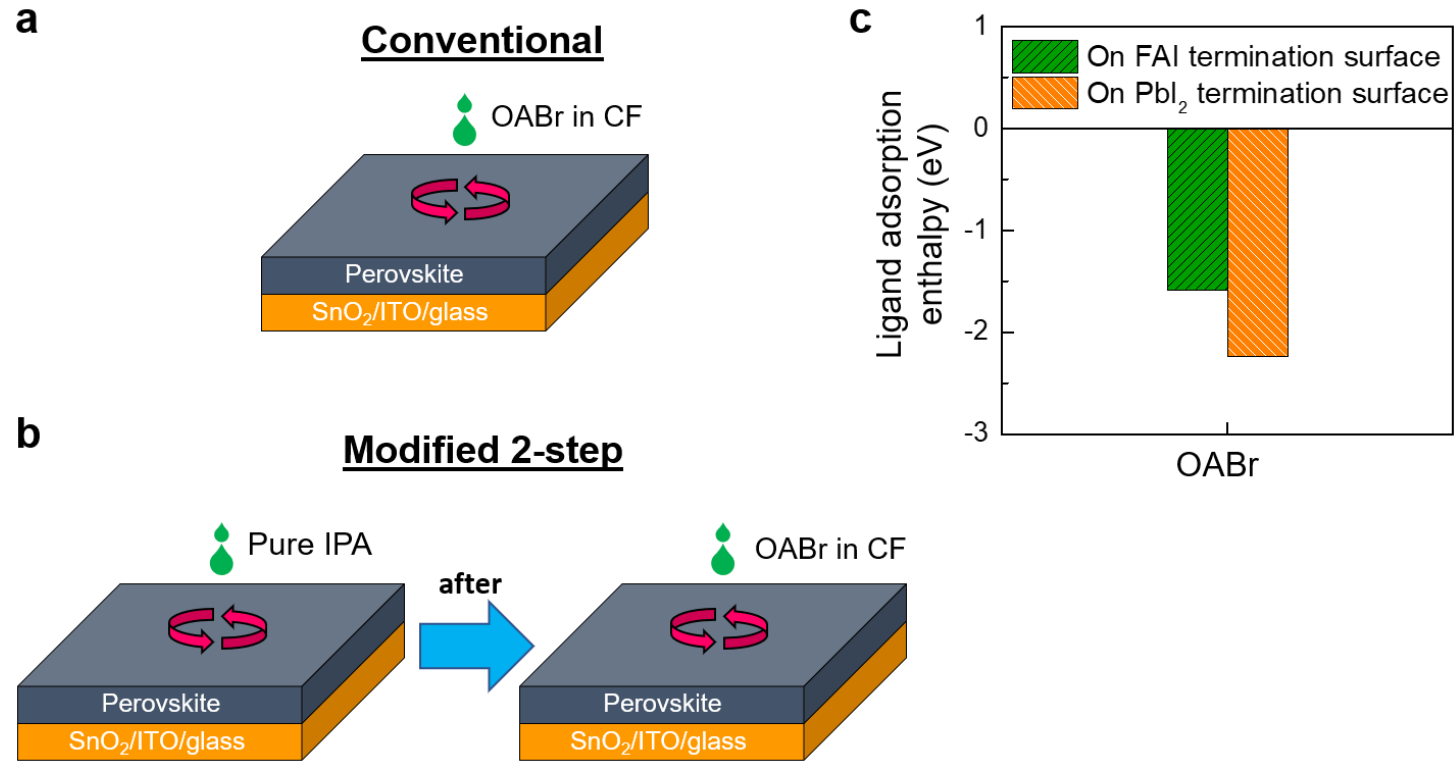

Figure S7. Modified surface treatment process. Schematic performance for the (a) conventional and (b) modified surface treatments procedures. (c) Enthalpy of adsorption of $\mathrm{OABr}$ on the surfaces.

a

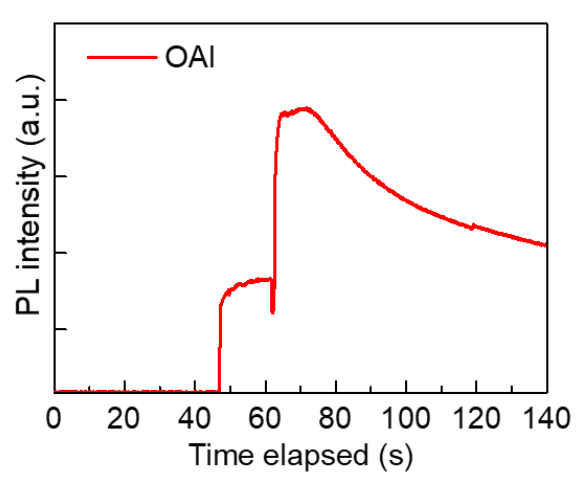

b

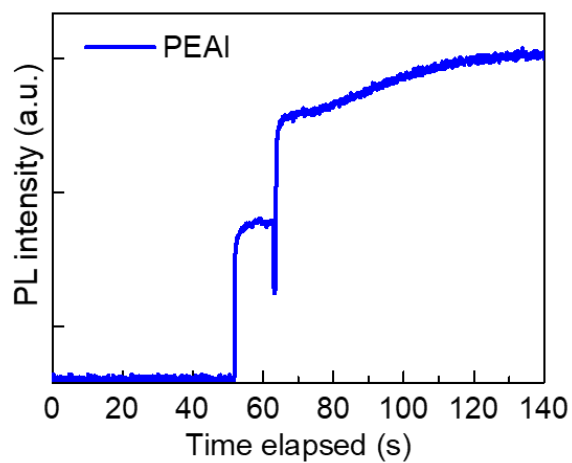

Figure S8. In situ PL of films undergoing surface treatment. Evolution of the PL intensity of films treated with (a) $10 \mathrm{mM}$ OAI or (b) $10 \mathrm{mM}$ PEAI as a function of time. 


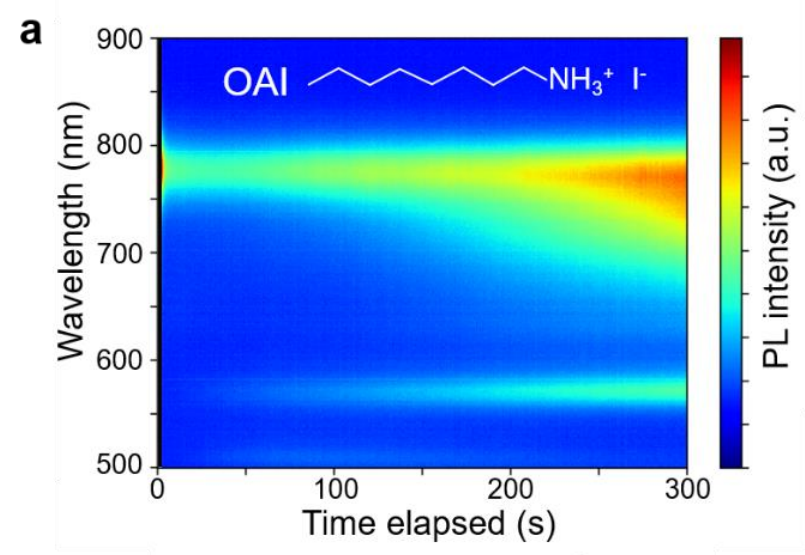

C

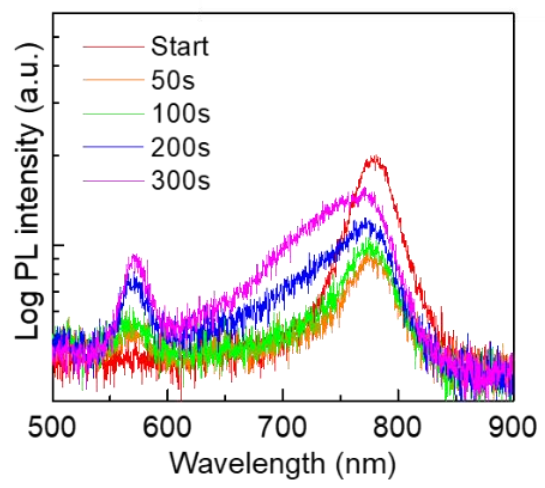

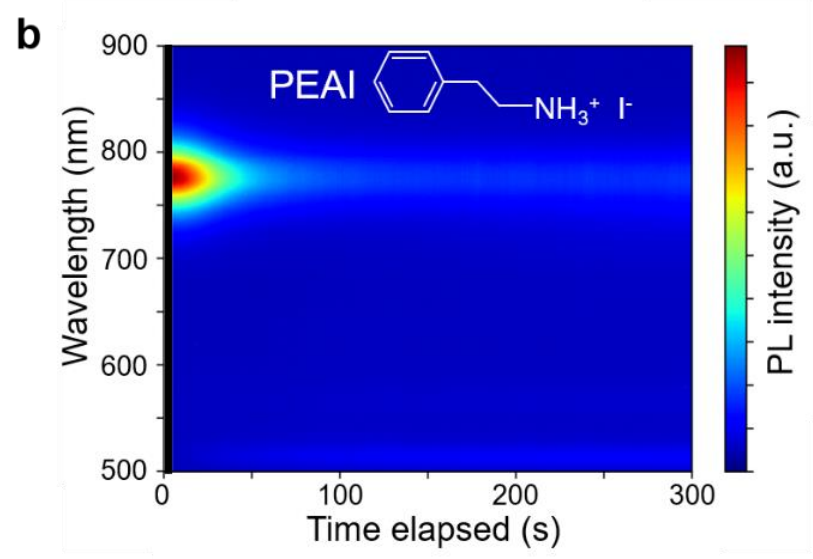

e

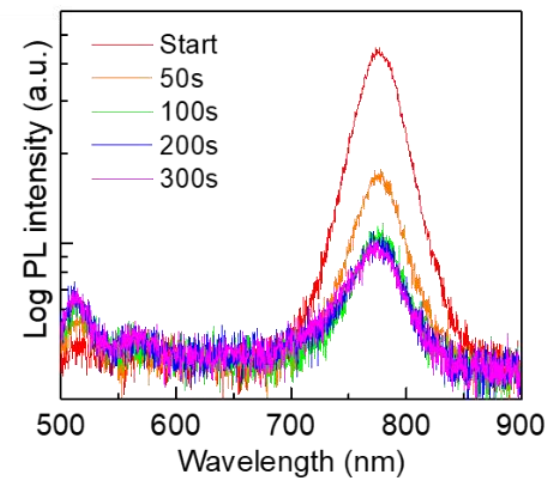

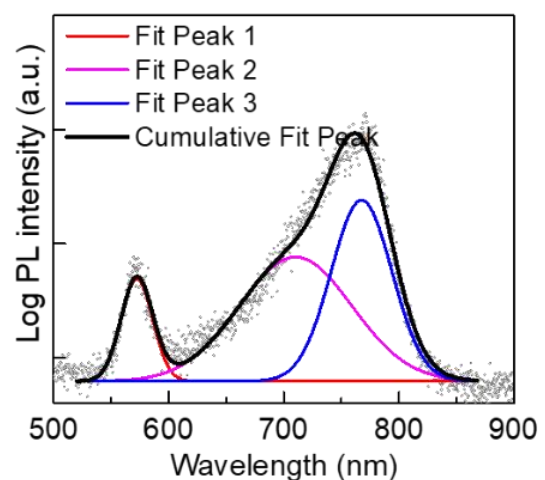

Figure S9. In situ PL of films undergoing post-annealing. Contour plots of perovskite films treated with (a) $25 \mathrm{mM}$ OAI or (b) $25 \mathrm{mM}$ PEAI in IPA undergoing post-annealing. Evolution with time of the raw PL spectra of the films treated with (c) $25 \mathrm{mM}$ OAI or (d) $25 \mathrm{mM}$ PEAI during post-annealing. (E) Deconvoluted peaks of the PL spectra at 300s for the OAI treated film.

\section{$\underline{\text { Supplementary References }}$}

1. Cullity, B.D., and Stock, S.R. (2001). Elements of X-Ray Diffraction, 3rd Edition, Ch. 410, Ch. 14-5.

2. Kato, M., Fujiseki, T., Miyadera, T., Sugita, T., Fujimoto, S., Tamakoshi, M., Chikamatsu, M., and Fujiwara, H. (2017). Universal rules for visible-light absorption in hybrid perovskite materials. J. Appl. Phys. 121, 115501. 